\title{
Processes Operative During Delay of Gratification ${ }^{1}$
}

\author{
J. Frank Yates ${ }^{2}$ and Glenda L. Revelle \\ The University of Michigan
}

Sixty-four middle-class preschool children chose between waiting for a preferred item and receiving a less desired item immediately. The items were of different classes, i.e., one food and one toy, and subjects waited with one, both, or neither of the rewards available for viewing. The likelihood that a subject would continue waiting for the delayed reward was found to increase as a function of previous waiting time rather than decrease or remain constant as required by two current theories of delay of gratification processes, an aversive affect model implied by Mischel and Ebbesen (1970) and Atkinson and Birch's (1970) "dynamics of action." Although display of rewards impaired successful delay, replicating previous results, dynamics of action predictions of differential effects for display of immediately available and delayed outcomes of different classes were not confirmed. Systematic observation of spontaneous subject activities during the delay period offered additional support for the notion that distraction facilitates successful waiting behavior. A decision-attention model is proposed to account for the present results as well as those of previous studies of delay of gratification.

In any delay of gratification situation, a person must choose between waiting for a delayed attractive outcome and taking a less desirable outcome that is available immediately. It has been suggested by Mischel (1974b) and others that there are actually two components to this decision process. First, the person must choose whether or not to begin waiting for the preferred

\footnotetext{
'We would like to thank the director, Mr. Jack Lewis, and the staff of the Red Bell Nursery of Plymouth, Michigan, for helping us obtain subjects for this study and providing access to their facilities. We are also grateful to Ms. Shirley St. Peter and Ms. Carolyn Jagacinski for their work as experimenters.

${ }^{2}$ Address all correspondence to $J$. Frank Yates, 136 Perry Building, Department of Psychology, University of Michigan, Ann Arbor, Michigan 48104.
} 
alternative. If he/she chooses to do so, he/she must then sustain that choice throughout the required waiting period. This study was designed to examine what happens during the second phase in the delay of gratification process - the psychological mechanisms operative during the period of time after the decision to wait has been made but before the preferred reward becomes available.

Based on the results of a series of studies (Mischel \& Baker, 1975; Mischel \& Ebbesen, 1970; Mischel, Ebbesen, \& Zeiss, 1972; Mischel \& Moore, 1973; Mischel \& Underwood, 1974), Mischel and his colleagues have proposed the currently dominant theory of processes operative during delay. Mischel's model is based on the premise that waiting for a desired outcome induces unpleasant frustration affect. Specifically, this aversive affect results from (1) the person's inability to obtain the preferred outcome immediately, (2) the conflict implicit in the choice situation, and (3) the mere activity of waiting. In addition to postulating the existence of frustration affect, Mischel asserts further that the strength of such affect is the governing factor in delay behavior. If the affect becomes too intense during the waiting period, the person will terminate his/her wait by taking the less attractive, immediately available item simply to escape from the situation and end the unpleasant feelings. Thus any activity during the waiting period that increases frustration affect should tend to decrease waiting times, whereas activities that decrease the aversive affect should facilitate waiting. To test this hypothesis, the Mischel group has manipulated the subject's attention to and thoughts about the outcomes in delay situations. The prediction that increased exposure to and thought about the outcomes should lead to decreased waiting times has been confirmed experimentally (Mischel \& Ebbesen, 1970; Mischel et al., 1972).

The role of aversive affect in the entire process, however, has simply been inferred rather than investigated directly. Since reminders of the delay situation have led to decreased delay times, it has been assumed that the intervening variable is increased frustration affect. The affect itself and its effects on behavior have been accepted without direct evidence. There have been some recent investigations concerning the relationship of affect to delay of gratification (Moore, Clyburn, \& Underwood, 1976; Schwarz \& Pollack, 1977); however, these studies have focused on the effect of the subject's affective state on his/her initial choice in a delay paradigm, not on the processes sustaining a delay once it has begun. The results of Mischel's most direct test of the effects of aversive affect, in fact, contradict the predictions of the model (Mischel et al., 1972). Children who were instructed to "think sad thoughts" during the delay period, presumably creating additional negative affect, did not terminate their waiting any sooner than children instructed to think about the rewards or children who were given no instruc- 
tion concerning their thoughts during the delay period. In each of these conditions subjects waited with both rewards present, so the base level of frustration affect should have been equivalent across the groups. The additional negative affect induced in the "think sad" group, then, did not increase the tendencies of the subjects in that group to terminate the delay period, as would be expected if aversive affect were the governing factor in delay behavior. Since the very existence of unpleasant affect during the waiting period and its instrumental role in delay processes form the foundation of Mischel's theory, these factors require a more direct substantiation than has previously been offered.

One implication of the central role of affect can be tested directly. According to the model, the activity of waiting, especially when combined with thoughts about the outcomes, induces aversive affect. So, as long as the person continues to wait, aversive affect should continue to exist also. If one interprets the model to say that aversive affect is a direct function of the amount of waiting or thought about the outcomes, then it follows that the longer the person waits, the higher his/her aversive affect level should become. A less stringent interpretation suggests that aversive affect remains constant as long as the person waits and/or thinks about the outcomes. At any rate, the model leads to clear-cut predictions about one observable aspect of waiting behavior: The likelihood that the person will continue to wait should be either constant or a decreasing function of the amount of time he/she has already waited.

As an alternative to Mischel's explanation of delay processes, Birch and Atkinson (1974) have applied their general theory of motivation, "the dynamics of action" (Atkinson \& Birch, 1970), to behavior in delay situations. Although the justification is different, Birch and Atkinson's theory leads to predictions about continued waiting that are similar to those of Mischel's theory. Within the dynamics of action framework, the behavior that occurs at any given time depends on the relative strengths of "tendencies" to engage in each of the possible behaviors available to the person; i.e., whichever tendency is strongest at a particular time will determine the person's behavior at that moment. The theory also asserts that the tendency for a given behavior decreases in a negatively accelerated fashion toward an asymptote during the time in which the person actually engages in that behavior. In addition, the strength of the tendency for a particular behavior increases as a linear function of the amount of time the person is exposed to an "instigating force" for the behavior. If the person neither engages in the behavior nor is exposed to an appropriate instigating force, the tendency for that behavior remains constant.

Two of the behaviors available to the person during the waiting period in a delay of gratification situation are continued waiting and terminating 
the delay period by accepting the less attractive outcome. According to the dynamics of action, as long as the person engages in waiting behavior, the tendency to wait will be continually decreasing. Eventually, the waiting tendency should be dominated by the tendency to terminate the delay situation. If the person is exposed to favorable consequences of terminating the delay, e.g., the immediately available outcome, the tendency for termination should overtake the tendency for waiting even sooner. Regardless, however, the probability that a person continues to wait in a delay situation should be a decreasing function of the amount of time he/she has already waited. Thus the first aim of the present study was to test these predictions of Mischel's and Birch and Atkinson's models by examining the pattern of waiting behavior terminations over a complete delay period.

Although the aversive affect and the dynamics of action models have similar implications concerning the effects of previous waiting time on continued waiting behavior, under certain conditions their predictions diverge regarding the effects of exposing the subject to his/her potential rewards during the waiting period. Mischel and Ebbeson's (1970) study illustrates the effects generally found. Subjects waited most successfully when neither the delayed nor the immediately available outcome was visible. There was no difference in the amount of time subjects delayed when exposed to either of the rewards alone. Subjects who could see both outcomes waited marginally significantly less time than did subjects. who saw displays of only one of the rewards. These results are congruent with the prescriptions of Mischel's model, which proposes that the display of either outcome should lead to frustration affect and therefore shortened waiting times. The model also specifies that the intensity of affect should be greatest, and thus waiting times shortest, when both the delayed and immediately available outcomes are displayed.

Birch and Atkinson (1974) demonstrate how the dynamics of action can account for the display effects found in the various studies of the Mischel group to date. Their analysis also suggests certain effects the aversive affect model does not predict. They indicate that the consequences of exposure to the delayed and immediately available outcomes generally should be quite different. In particular, displaying the immediately available outcome should inhibit effective delay behavior much more than displaying the delayed outcome. It is claimed that this result has not been found in Mischel's previous studies because the delayed and immediately available outcomes in those studies have always been members of the same class (e.g., both foods).

Birch and Atkinson's argument is as follows. First, they specialize the term waiting to apply only when the subject is actually thinking about and anticipating the delayed outcome during the waiting period. A display of 
the delayed outcome is likely to lead to a great deal of conscious waiting during the delay period, thus causing the tendency for (further) waiting to decline toward its asymptote. On the other hand, displaying the immediately available outcome amounts to providing an instigating force for terminating the delay period. Since tendencies for behaviors generally increase in response to instigating forces more rapidly than they decrease toward their asymptotes when the behaviors are actually taking place, exposure of the immediately available outcome should lead to shorter delay times than exposure of the delayed outcome. Now, if both outcomes happen to be in the same class, "displacement" should occur such that a display of the delayed outcome not only leads to conscious waiting but, more importantly, induces an instigating force for terminating the delay period. The role of displacement can be illustrated as follows. Suppose a child is offered a choice between a pretzel now and a marshmaliow, which he/she prefers, later. If the child becomes hungry while waiting for and thinking about the marshmallow, he/she can satisfy his/her hunger at least partially by taking the immediately available pretzel. This logic applies even more directly to cases in which the delayed reward is simply a larger quantity of the immediately available outcome, e.g., two pretzels versus one pretzel. So, in this case, displaying any of the rewards increases the subject's tendency to take the immediately available one. This uniform display effect should not be expected when the outcomes are qualitatively different from each other.

So, a second aim of the present research was to compare the viability of Mischel's and Birch and Atkinson's claims by exposing subjects in delay of gratification situations to outcomes from two distinct classes. Both models imply that delay behavior should be most successful when the subject is exposed to neither outcome. While the aversive affect model predicts no difference in the delay behavior of subjects exposed to either the delayed or the immediately available outcome alone, the dynamics of action model implies that display of the delayed outcome should impair effective waiting less. Both models predict that waiting behavior should be least successful, markedly so according to the aversive affect model, when the subject is exposed to both outcomes.

Since the focus of this study was on processes occurring during the waiting period in delay situations, we also observed the behaviors of subjects during that period. Casual observations of subjects' waiting period activities have been reported by Mischel (1974a, for example). The present study simply included a more detailed and systematic procedure for observing and recording subjects' spontaneous activities while waiting. These observations provide an additional source of evidence regarding the status of current theories, an opportunity to determine the types of behavior that are associated with successful delay, and the possibility of suggesting new 
waiting strategies that have not been included in contemporary theories of delay behavior.

\section{METHOD}

\section{Subjects}

Subjects in this study were middle-class children from two private preschools. There are two reasons why preschool children were chosen to be the subjects in this study. First, small units of time are more salient to young children than to adults, so it is more feasible to offer a meaningful delay choice to this group within an experimental paradigm. Second, we were interested in testing predictions arising from Mischel's model and making comparisons with the results of his studies in which he has used preschool subjects. There were 64 subjects in this study. An additional 6 subjects were dropped due to refusal to come for the second session or failure to understand the instructions. Ages ranged from 38.5 to 69 months, with a mean age of 53.5 months. There were equal numbers of males and females in each of the four conditions, and mean age was equated across conditions. The two experimenters were both female; one conducted the preliminary session for each child and the other conducted the experimental session. The experimenter who conducted the experimental session was naive with respect to the issues and hypotheses of the study.

\section{Preliminary Session}

In order to select the items to be offered to a given child in the delay situation, a preliminary session was conducted to determine his/her preferences among the available items. Each subject was told that he/she would be shown a variety of things in order to determine which ones he/she liked best. First, all of the items (a finger puppet, a whistle, a party favor blow-out, a small box of raisins, several marshmallows, and several animal crackers) were presented. Each item was named, and the toys were demonstrated; then all 15 of the possible pairings of the six items were presented in a standard, random order, with left/right position randomized also. For each pair of items, the subject was asked to point to the item he/she "liked best." After completing the 15 choices, the subject was told that there would be another session the following day.

Based on the individual child's preferences, one food item and one toy were chosen to be offered to that child in the experimental session. Using $X$ 
to represent the preferred item for which the child had to wait and $Y$ to represent the less preferred item which the child was allowed to have immediately, the items to be used were determined according to the following criteria: (1) Item $X$ and Item $Y$ were of different classes (one food and one toy). (2) Item $X$ was preferred to Item $Y$ in the direct pairing of these two items. (3) In at least one paired comparison, one other item (of either class) was preferred to Item $X$; similarly, Item $Y$ was preferred to some other item (of either class) in at least one paired comparison. (4) The difference between the numbers of items to which Items $X$ and $Y$ were preferred was maximal, given the other criteria. Restrictions 3 and 4 were included to ensure a moderate, yet substantial, discrepancy in the subject's preference of Item $X$ over Item $Y$. The preferred item was food for half of the subjects in each condition and a toy for the other half.

\section{Experimental Session}

In order to equate the subject's hunger level in the preliminary and experimental sessions as closely as possible, each subject's sessions were scheduled at the same time of day on 2 consecutive days. For the delay session, subjects were taken to a small room in an experimental trailer. In the room there were a table and one chair, a fan blocked from view by a partition, one window covered with blinds, and two one-way mirrors. The child was seated at the table, facing a wall. After talking to the child for several moments to help him/her become comfortable in the room, the experimenter initiated a game with the child to teach him/her a knocking procedure for signaling the experimenter to return from an adjacent room. She told the child: "I'm going to go into the next room and close the door. After I have left, I want you to knock on the table two times like this Idemonstrates]. When you knock on the table two times I will come right back! Okay?" This procedure was repeated several times, until the child understood that he/she could "bring the experimenter back" by knocking.

The experimenter then presented the child's preferred and less preferred items and asked the subject which one he/she wanted, to confirm his/her preference from the previous day. If the child switched his/her preference, which rarely happened, the remainder of the session was conducted on the basis of the newly declared preference. From this point on, the procedure described by Mischel and Ebbesen (1970) was followed. The experimenter told the child that she was going to leave the room, and that if the child waited for her to come back he/she could have his/her preferred reward. The child was told that if he/she did not wish to wait, he/she could use the knocking procedure and the experimenter would come back, but the child would then receive his/her less preferred reward. 
Each subject waited in one of four display conditions: (1) both items present in front of the subject during the waiting period, (2) preferred item present, (3) less preferred item present, or (4) neither item present. The experimenter placed the item(s) to be displayed on the table in front of the child and placed the other(s) in a cake tin, to be left beneath the table. She then repeated the contingencies and left the room. If the subject did not signal for the experimenter to return, she waited in the adjacent room for 20 minutes. During the waiting period, the experimenter recorded the subject's behaviors within each 60-second interval. After the subject signaled or 20 minutes had passed, the experimenter returned to the room and gave the child the appropriate reward. Each subject was then given a few minutes in which to eat or play with his/her reward before being taken back to the classroom.

\section{RESULTS}

The processes governing the initial decision between the delayed and immediately available outcomes may be qualitatively different from those responsible for the maintenance of waiting once it has been chosen. Since this study focuses on delay-sustaining processes, the analyses reported here include only the data of subjects who exhibited at least minimal waiting behavior, i.e., did not take the less preferred item immediately. There were 7 subjects, distributed across the conditions, who chose to terminate the session immediately; thus, analyses were performed on the data of the remaining 57.

\section{Continued Waiting Probability Function}

The probability that a subject continues waiting (W), given previous waiting time $(t)$, may be represented by $\mathrm{P}(\mathrm{W} \mid t)$. Strictly speaking, "continued waiting"' is an instantaneous phenomenon. Practically, however, an observer can judge that a person has continued to wait at a certain instant in time only if the person is still waiting at some point beyond that instant. Our procedure for estimating the $\mathrm{P}(\mathrm{W} \mid t)$ function involved partitioning the 20 -minute waiting period into 1-minute intervals. A subject was considered to have "continued waiting" at a particular moment if at the end of the next 1-minute interval he/she was still waiting. Thus, given a number of subjects who have waited 5 minutes, for example, some may continue waiting through the 6th minute while others may terminate the waiting period before the end of the 6th minute. The former group is judged to have con- 


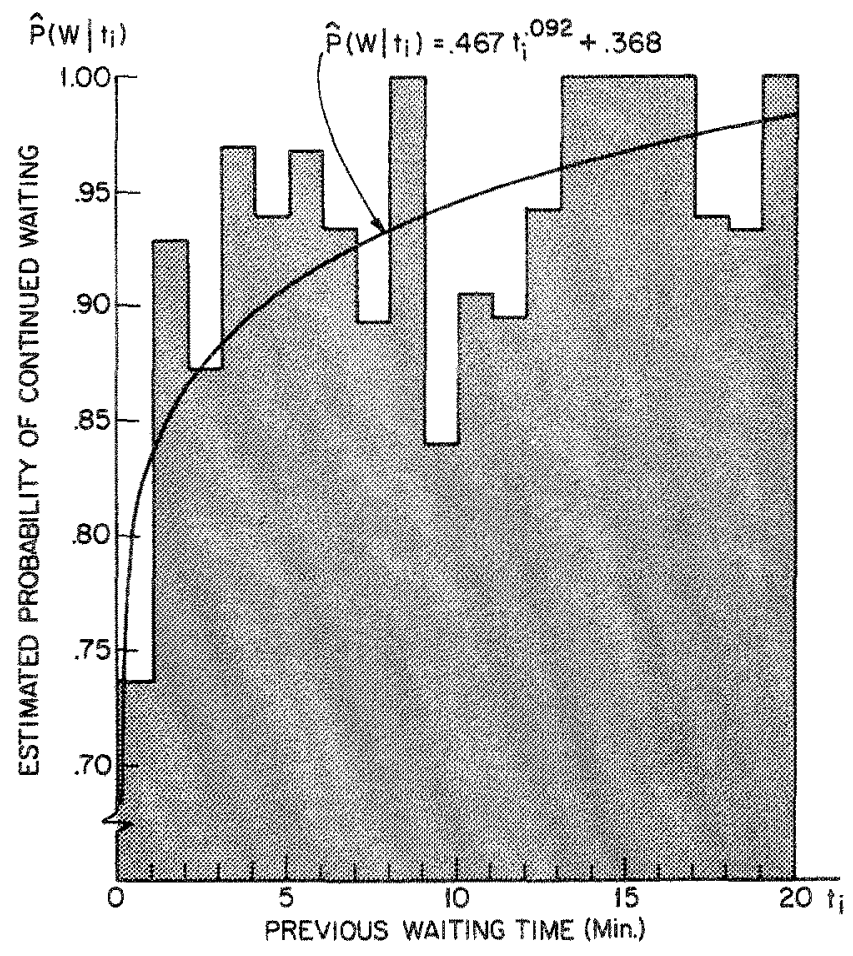

Fig. 1. Estimates of the probability of continued waiting as a function of previous waiting time.

tinued waiting, given 5 minutes of previous waiting time. In this way, it was determined for every 1 -minute point what proportion of the remaining subjects continued to wait, and analyses were carried out to determine whether and how the proportion of subjects continuing to wait depended on previous waiting time.

Figure 1 displays the successive values of these proportions. There is no indication that probabilities of continued waiting decline as a function of previous waiting time, as the dynamics of action and the strong form of the aversive affect model imply they should. There is also no evidence for the assertion of the weak form of the aversive affect model that probabilities of continued waiting should be constant. Indeed, there is a clear tendency for continued waiting probabilities to increase with time. These observations are corroborated by the results of regression analyses. The best-fitting linear regression function has a positive slope of $.006(F(1.18)=6.91, p<.05)$. The increasing curvilinear function shown in the figure, $\hat{\mathrm{P}}\left(\mathrm{W} \mid t_{\mathrm{i}}\right)=.467 \hat{t}_{\mathrm{i}}^{\mathrm{6} 2}$ 
+.368 , fits even better. The correlation between actual and predicted values of $\hat{\mathrm{P}}\left(\mathrm{W} \mid t_{\mathrm{i}}\right)$ for that function is $.64(t(18)=3.54, p<.005)$.

\section{Outcome Display Effects}

Since the distribution of waiting times tended toward bimodality, results were evaluated through the use of contingency table analyses. There were no differences in waiting behavior between subjects whose preferred outcome was food and those who preferred toys. The analyses were therefore performed ignoring preferred item class distinctions.

Table I contains the frequencies of subjects in each display condition who either waited for the delayed outcome for the full 20-minute period or terminated their waiting early. Subjects exposed to no outcome display were relatively less inclined to terminate waiting early than were subjects exposed to at least one of the outcomes ( $p<.05$, Fisher exact probability test). Thus the data are consistent with previous indications that outcome displays induce processes that inhibit waiting behavior. The analysis provides no indication that any outcome display condition interferes with successful waiting behavior more than the other display conditions. Thus the results do not provide support for the dynamics of action claim that when the items are from different classes, displays of immediately available and delayed outcomes should affect waiting behavior differently. There is also no evidence for the prediction of the aversive affect model that successful delay behavior should be substantially less likely to occur when both outcomes are exposed than when only one item is displayed.

\section{Waiting Period Activities}

Observations of subject behaviors during the waiting period were recorded in 1-minute time intervals. Thus only the observations for the 42

Table I. Frequencies of Categories of Waiting Behavior by Display Condition

\begin{tabular}{lcc}
\hline & \multicolumn{2}{c}{ Waiting } \\
\cline { 2 - 3 } Displayed outcome & Partial & Full \\
\hline Immediate & 12 & 3 \\
Delayed & 12 & 2 \\
Both & 12 & 3 \\
Neither & 7 & 6 \\
\hline
\end{tabular}


subjects who waited at least 1 minute were analyzed. Each activity was coded dichotomously for its occurrence or nonoccurrence during a given subject's waiting period. The resulting $2 \times 2$ contingency tables for each activity (Partial-Full Wait $X$ Activity Occurrence-Nonoccurrence) were eXamined for association via Fisher exact probability tests. Subjects who waited the full delay period were much more likely to (a) talk to themselves about things not related to the delay situation $(p<.05)$, (b) yawn $(p<.001)$, (c) rest their heads on their arms $(p<.05)$, (d) look around at the floor of the room $(p<.005)$, and (e) fidget in their chairs $(p<.05)$. On the other hand, subjects who did not wait through the entire delay period were much more prone to (a) silently tap the table in rehearsal of the signal for terminating the delay period $(p<.001)$ and (b) stare at the prizes $(p<.05)$. Other behavioral categories in which there were no differences between subjects who terminated early and those who waited were (a) talking to themselves about things related to the delay situation, (b) touching the prizes, and (c) creating irrelevant games to play while waiting.

\section{DISCUSSION}

The results provide no support for Atkinson and Birch's dynamics of action theory as an explanation for delay of gratification behavior. Although the current study does provide additional evidence consistent with Mischel's suggestion that distraction from the particulars of the delay situation facilitates waiting, the results are incompatible with the major thesis of Mischel's aversive affect model-that induced frustration affect is the governing factor in delay behavior. In light of the lack of support for these two leading theories of delay behavior, the major task of providing a coherent and parsimonious accounting for that behavior remains.

An extension of basic principles of decision theory seems capable of explaining delay behavior rather well. Most theories about decisions involving outcomes receivable at different times (cf. Fishburn, 1970, Chap. 7; Jamison, 1970) suggest that the crucial issue is the trade-off between a value differential and a time differential. In a delay of gratification situation, the immediately available outcome is valued less than the delayed outcome. The difference in subjective value is the value differential. On the other hand, the amount of time that must elapse before the delayed outcome is receivable is obviously greater than the time before the immediately available outcome can be received. The person's perception of that difference in time is the time differential. In order for the person to choose to wait for the delayed outcome, he/she must (implicitly) judge the magnitude of the value differential to more than offset the magnitude of the time dif- 
ferential. Otherwise, it is not "worth it" for the subject to wait. Clearly, this logic implies that if a person is inclined to wait when the time differential is 20 minutes, he/she should be even more willing to wait when the time differential is 15 minutes. Thus one would expect precisely the kind of pattern of results observed in the present study; probabilities of continued waiting should increase as a function of the amount of time the person has already waited since the time differential becomes progressively shorter the longer the person waits.

What about distraction? At any time during a delay period, the person can do one of several things. First, he/she could recognize that he/she has an opportunity to make a decision about waiting. He/she can then either decide to continue waiting or decide to terminate the delay period. The person might not recognize that a decision opportunity exists at all if he/she happens to be thinking about things other than the delay situation. The net effect of such distraction is continued waiting, but by default rather than by deliberate decision. When a delay period begins, the opportunity for making a decision should be very salient to the person. And given the relatively large time differential during the early stages of the delay period, delay terminations should be very common. Once the delay period has begun in earnest, there should be a greater chance that the person will become distracted, especially if there are few reminders of the delay situation physically present. The results reported here are certainly compatible with this viewpoint.

The data of the present investigation, as well as those of previous studies of delay of gratification, can be explained very readily by the proposed "decision-attention model." A couple of recent studies illustrate the ability of the model to integrate a variety of results in the area. Corfield, Al-Issa, and Johnson (1976) asked each of their subjects to work a crossword puzzle while waiting for a delayed reward. For half the subjects the words making up the puzzle were relevant to the awaited reward, whereas for the other half they were irrelevant. For groups whose reward was not contingent on the work they did, as is generally the case in delay paradigms, subjects waited significantly longer when puzzle words were irrelevant to the outcomes in the delay situation. As suggested by the decision-attention model, subjects exposed to the delay-irrelevant words may well have not recognized that a delay decision opportunity existed. Miller and Karniol (1977) found that when subjects devalued the preferred reward in a delay situation, they generally had shorter delay times as well. This outcome is accommodated within the decision-attention model in that devaluation of the preferred reward amounts to a reduction of the value differential. Miller and Karniol's study also suggests that one of the things that may explain various delay phenomena is reassessment of value differentials as well as time differentials during the delay period. 
It is possible that delay behavior is determined by the components of the decision-attention model and constructs such as aversive affect. At the present time, however, there are no results reported in the literature that seem to demand the role of such auxiliary constructs. Nevertheless, future research should not only be directed toward systematic and thorough testing of the decision-attention model as here stated but should also cxamine elaborations of the model that might incorporate concepts like aversive affect.

\section{REFERENCES}

Atkinson, J. W., \& Birch, D. The dynamics of action. New York: Wiley, 1970.

Birch, D., \& Atkinson, J. W. Comments on the discussion. In B. Weiner (Ed.), Cognitive views of human motivation. New York: Academic Press, 1974. Pp. 71-84.

Corfield, V., Al-Issa, I., \& Johnson, B. Effects of verbal cues on the delay of self-gratification. Journal of Psychology, 1976, 94, 167-171.

Fishburn, P. C. Utility theory and decision-making. New York: Wiley, 1970.

Jamison, D. Studies in individual choice behavior. RAND Memorandum No. P-4255. Santa Monica, California: RAND Corporation, 1970.

Miller, D. T., \& Kamiol, R. Children's cognitive re-evaluations in a self-control situation: $A$ test of the self-persuasion hypothesis. Paper presented at the biennial meeting of the Society for Research in Child Development, New Orleans, March 1977.

Mischel, W. Cognitive appraisals and transformations in self-control. In B. Weiner (Ed.), Cognitive views of human motivation. New York: Academic Press, 1974. Pp. 33-49. (a)

Mischel, W. Processes in delay of gratification. In L. Berkowitz (Ed.), Advances in experimental social psychology (Vol. 7). New York: Academic Press, 1974. (b)

Mischel, W., \& Baker, N. Cognitive appraisals and transformations in delay behavior. Journal of Personality and Social Psychology, 1975, 31, 254-261.

Mischel, W., \& Ebbesen, E. B. Attention in delay of gratification. Journal of Personality and Social Psychology, 1970, 16, 329-337.

Mischel, W., Ebbesen, E. B., \& Zeiss, A. Cognitive and attentional mechanisms in delay of gratification. Journal of Personality and Social Psychology, 1972, 21, 204-218.

Mischel, W., \& Moore, B. Effects of attention to symbolically presented rewards on selfcontrol. Journal of Personality and Social Psycholugy, 1973, 28, 172-179.

Mischel, W., \& Underwood, B. Instrumental ideation in delay of gratification. Child Development, 1974, 45, 1083-1088.

Moore, B. S., Clyburn, A., \& Underwood, B. The role of affect in delay of gratification. Child Development, 1976, 47, 273-276.

Schwarz, J, C., \& Pollack, P. R. Affect and delay of gratification. Journal of Research in Personality, 1977, 11, 147-164. 